\title{
Influence of structure to plastic deformation resistance of alumi- num alloy 1560 after groove pressing treatment
}

\author{
E.N. Moskvichev ${ }^{\dagger}$, V.A. Skripnyak, V.V. Skripnyak, A.A. Kozulin, D.V. Lychagin \\ †em_tsu@mail.ru
}

The National Research Tomsk State University, Tomsk, Lenina ave. 36, 634050 Tomsk, Russia

As a result of groove pressing treatment the influence of structural changes was investigated in the samples made of rolled sheet of an aluminum alloy 1560 in the presented research work. Mechanical properties of material was investigated experimentally in quasi-static uniaxial tensile tests at a speed of deformation of $\mathrm{s}^{-1}$ in the as-received condition and after severe plastic deformation by groove pressing technique. Change of microhardness value of rolled sheet $1.5 \mathrm{~mm}$ in thickness has been determined in a sample after four cycles of groove pressing in comparison with a sample in as-received condition. It was determined that after four treatment cycles, yield strength and fracture resistance of rolled alloy sheet increased in 1.4 and 1.5 times, respectively, and microhardness value increased in 2.7 times. Hardening of the studied alloy was accompanied by relative reduction of ultimate strength deformation from $21 \%$ in a sample made from aluminum alloy 1560 in as-received condition to $16.7 \%$ in a sample made of aluminum alloy 1560 after four groove pressing cycles. Analysis of change results in the grain structure was made by electron backscatter diffraction method. Groove pressing technique processing of aluminum alloy 1560 leads to the formation of bimodal grain structure, which consists of coarse elongated grains and aggregates of micron and submicron sizes with an equiaxial shape. The obtained data are consistent with the experimental data of strength characteristics increase of aluminum alloy 1560 by comparison with other methods of severe plastic deformation.

Keywords: aluminum alloy, severe plastic deformation, mechanical properties, grain structure, groove pressing.

\section{Влияние структуры на сопротивление пластической дефор- мации алюминиевого сплава 1560 после обработки методом прессования рифлением}

\author{
Москвичев Е.Н. ${ }^{\dagger}$ Скрипняк В.А., Скрипняк В.В., Козулин А.А., Лычагин Д.В. \\ †em_tsu@mail.ru
}

Национальный исследовательский Томский государственный университет, пр. Ленина 36, 634050 г. Томск, Россия

В представленной работе исследовали влияние структурных изменений в образцах, изготовленных из листового проката алюминиевого сплава 1560, в результате обработки методом многократного прессования рифлением. Механические свойства материала в состоянии поставки и после четырех циклов обработки интенсивной пластической деформацией методом прессования рифлением были исследованы экспериментально при одноосном квазистатическом растяжении при скорости деформации $1 \mathrm{c}^{-1}$. Было определено значение изменения величины микротвердости листового проката 1.5 мм толщины в образце после четырех циклов прессования рифлением в сравнении с образцом в состоянии поставки. Установлено, что после четырех циклов обработки листового проката сплава, условный текучести и временное сопротивление разрушению возрастают в 1.4 и 1.5 раз, соответственно, а величина микротвердости возрастает в 2.7 раза. Эффект упрочнения исследуемого сплава сопровождается относительным уменьшением деформации на пределе прочности с $21 \%$ в образце из сплава 1560 в состоянии поставки до $16.7 \%$ в образце из сплава 1560 после четырех обработки прессованием рифлением. Представлены результаты анализа изменений зеренной структуры, проведенного методом дифракции отраженных электронов. Обработка алюминиевого сплава 1560 методом прессования рифлением приводит к формированию бимодальной зеренной структуры, состоящей из крупных зерен вытянутой формы и скоплений зерен микронных и субмикронных размеров, имеющих равноосную форму. Полученные данные согласуются с экспериментальными данными о повышении прочностных характеристик алюминиевого сплава 1560, при использовании других методов интенсивной пластической деформации.

Ключевые слова: алюминиевый сплав, интенсивная пластическая деформация, механические свойства, зеренная структура, прессование рифлением. 


\section{1. Введение}

Повышенный интерес к обработке легких сплавов на основе алюминия, магния, титана методами интенсивной пластической деформации (ИПД) обусловлен возможностью комплексного повышения механических свойств за счет структурных изменений [1-6]. В этой связи представляют интерес возможности изменений структуры и прочностных свойств тонколистового проката легких сплавов с помощью нового метода ИПД прессования рифлением (ПР) [7-10]. Указанный метод был предложен для формирования ультрамелкозернистой зеренной структуры листового проката легких сплавов. В отличии от других технологий ИПД метод ПР позволяет формировать мелкозернистую структуру в пластинах, размер которых ограничен только производственными мощностями [7]. Ранее метод ПР был успешно использован для повышения прочностных характеристик тонколистового проката сплавов коммерчески чистого алюминия, магния, титана, меди, никеля и низкоуглеродистой стали [8-12].

В данной работе впервые проведено исследование модификации зеренной структуры и изменений механических свойств алюминиевого сплава 1560 (АМг6) в листовом прокате 1.5 мм толщины в результате многократного ПР.

Основной целью исследования было изучение влияния изменений в зеренной структуре сплава 1560 в результате ПР на закономерности деформации, прочностные характеристики и микротвердость.

\section{2. Описание методики прес- сования с рифлением}

В методе ПР интенсивная пластическая деформация развивается в зонах сдвига-изгиба плоского образца, зажатого между двумя пресс-формами, имеющими пазы трапециевидной формы (рис. 1). Как правило, образец подвергается нескольким циклам прессования. Один цикл прессования состоит из нескольких этапов:

(a - b) - деформация сдвига-изгиба в зонах образца, зажатого между двумя рифлеными пресс-формами плоского образца; (c) - деформация при выпрямлении рифленого образца путем обжатия между двумя плоскими пластинами; (d) - поворот образца на угол $180^{\circ}$ относительно нормали к поверхности образца; (е) - повторная деформация сдвига-изгиба ранее недеформированной области пластины; (f) - повторная деформация при выпрямлении.

Исходя из аналитической оценки после 4 циклов прессования обеспечивается накопление пластической деформации $\varepsilon_{\text {eff }}=4.64$ [13].

В работе применялась прессформа площадью $20 \times 120$ мм, с шагом рифления 2 мм и углами наклона плоскости $45^{\circ}$. Прессование выполнялось при начальной температуре $523 \pm 5 \mathrm{~K}$; скорости прессования - 10 мм/мин; максимальной нагрузке до 50 кН. Применялся режим циклического прессования - паузы (длительностью 1 мин). Для минимизации трения использовали высокотемпературную смазку на основе дисульфида молибдена.

\section{3. Методы исследования}

Образцы размером $120 \times 20 \times 1.5$ мм для прессования вырезались вдоль и поперек направления проката из листа алюминиевого сплава 1560 в состоянии поставки размером $2000 \times 2000 \times 1.5$ мм. Сплав 1560 имел следующий химический состав: $\mathrm{Mg}-6.124 \%, \mathrm{Mn}-0.598 \%$, $\mathrm{Fe}-0.351 \%$, Si $-0.310 \%, \mathrm{Zn}-0.203 \%$, Ti $-0.084 \%$, $\mathrm{Cu}-0.086 \%, \mathrm{Al}-$ остальное.

Измерялась микротвердость сплава в состоянии поставки и после 4 циклов прессования. Измерения микротвердости Нv проводились в соответствии с ГОСТ 9450-76 с использованием автоматического микротвердомера Instron Wilson Hardness Tukon 2500-6 с нагрузкой на индентор 50 г, выдержке при индентировании 5 секунд. Подготовку поверхности образцов перед измерением микротвердости проводили по стандартной методике с использованием шлифования и полировки до зеркальной бездефектной поверхности, до чистоты $\mathrm{R}_{\mathrm{z}}=0.1$ мкм.

Проведены испытания образцов в состоянии поставки и после $4 \mathrm{x}$ кратного ПР на растяжение в соответствии с ISO 6892_2009-08. Испытания по одноосному растяжению образцов проводили с использованием электромеханической испытательной системы Instron 5948 при скорости деформации $1 \mathrm{c}^{-1}$. Применялись образцы в виде плоских двухсторонних лопаток с размерами: длина рабочей части 7 мм; ширина 3 мм; толщина 1.4 мм и 1.5 мм радиус скругления, вырезанные поперек оси пластин электроэрозионным методом. Поверхности каждого перед испытанием шлифовалась до снятия видимых дефектов и царапин.

Исследования зеренной структуры проводили методом дифракции отраженных электронов (ДОЭ) на электронном микроскопе Tescan Vega II LMU. Поверхность образцов для исследования была подготовлена методом ионного травления на установке ION SLICER EM-09100. Анализ полученных данных проводился на лицензионном программном обеспечении HKL-Channel 5.

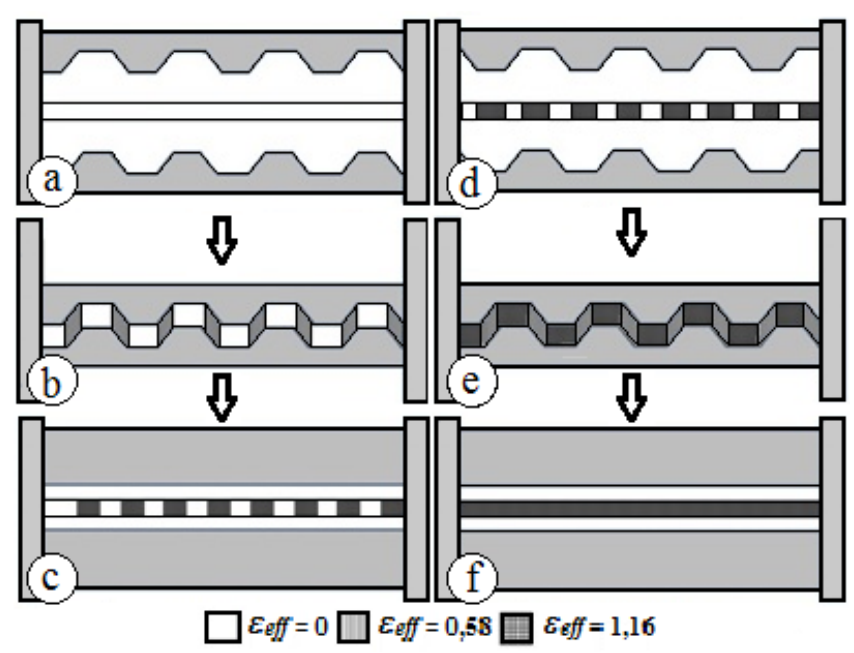

Рис. 1. Схема метода прессования с рифлением.

Fig. 1. Groove pressing process scheme. 


\section{4. Результаты и обсуждение}

Зеренная структура алюминиевого сплава 1560 в состоянии поставки характеризуется распределением размера зерна в диапазоне 6-54 мкм. Целесообразно выделение трех частичных диапазонов изменения характерных размеров зерен: 300 нм $<d<2.5$ мкм, 2.5 мкм $<d<10$ мкм, 10 мкм $<d<54$ мкм. Среднее значение размера зерна $-d_{\text {ср }}=12$ мкм. Возле крупных зерен наблюдаются более мелкие зерна (Рис. 2, а). Количество крупных зерен $(d>10$ мкм) немного больше количества средних зерен (2.5 мкм $<d<10$ мкм) (Рис. 2, b).

Зеренная структура алюминиевого сплава 1560 после четырех циклов прессования рифлением характеризуется распределением размеров зерен в диапазоне 2-40 мкм. Зерна с размерами 10 мкм $<d<54$ мкм имеют вытянутую форму, что является следствием воздействия интенсивной пластической деформации. Зерна с размерами 300 нм $<d<10$ мкм, имеют круглую форму, что свидетельствует о процессах рекристаллизации (Рис. 3, a).

В результате обработки сплава методом многократного ПР может быть достигнуто не только уменьшение среднего размера зерна, но и формирование
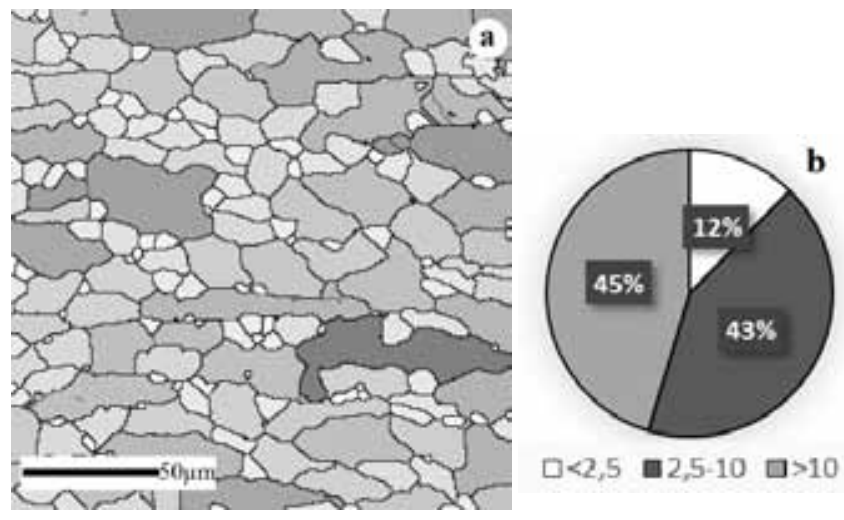

Рис. 2. а) Зеренная структура алюминиевого сплава 1560 в состоянии поставки; b) Круговая диаграмма количественного распределения размеров зерен.

Fig. 2. a) 1560 alloy untreated sample grain structure; b) Grain size quantitative distribution diagram.

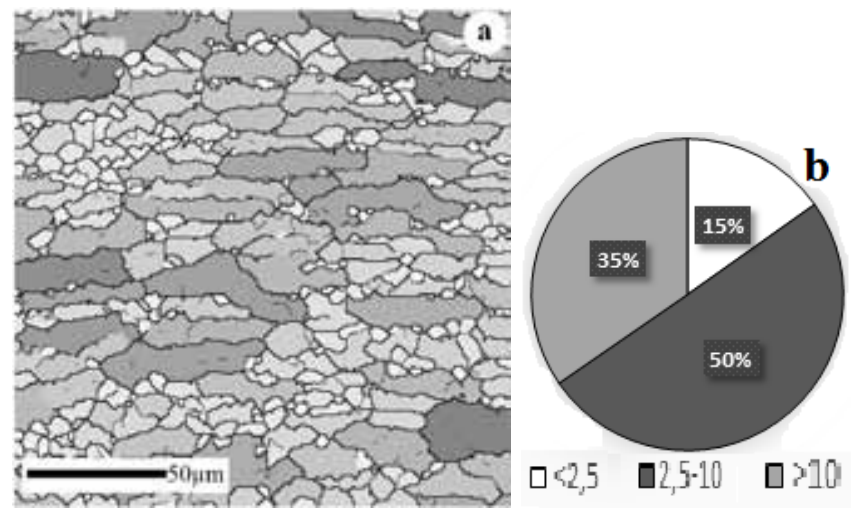

Рис. 3. а) Зеренная структура алюминиевого сплава 1560 после четырех циклов ПР; b) Круговая диаграмма количественного распределения размеров зерен, $\mu \mathrm{m}$.

Fig. 3. a) 1560 alloy treated sample grain structure; b) Grain size quantitative distribution diagram. бимодального распределения зерен по размерам. Относительное количество зерен с размерами $d<2.5$ мкм и 2.5 мкм $<d<10$ мкм увеличивается с увеличением количества повторных циклов прессования. Увеличение относительного количества зерен, превышающих 2.5 мкм, по сравнению с ультрамелкими зернами, может быть свидетельством протекания процессов динамической рекристаллизации в ходе прессования при повышенной температуре (Рис. 3).

Таким образом, обработка алюминиевого сплава 1560 методом прессования с рифлением листового проката позволяет получать в объеме материала зеренную структуру, состоящую из вытянутых крупных зерен и скоплений зерен микронных и субмикронных размеров. Аналогичная зеренная структура в алюминий-магниевых сплавах была ранее обнаружена после ИПД в работе [14].

После четырех циклов ПР микротвердость алюминиевого сплава 1560 увеличилась в среднем в 2.7 раза и достигла средних значений $\mathrm{HV}=112$. Микротвердость сплава в состоянии поставки не превышала HV = 41. Полученные данные согласуются с результатами двукратного повышения микротвердости алюминиевого сплава АА3003 после ПР, полученными Khodabakhshi с соавторами [15].

На рис. 4 показаны полученные диаграммы «условное напряжение - инженерная деформация» при одноосном растяжении плоских образцов алюминиевого сплава 1560 в состоянии поставки (а) и после четырех циклов ПР (b). Прочностные характеристики алюминиевого сплава 1560, приведенные в таблице, свидетельствуют об $38 \%$ увеличении условного предела текучести и $52 \%$ увеличения временного сопротивления разрушению в квазистатических условиях нагружения после обработки ПР. Эффект упрочнения сопровождается уменьшением предельной деформации до разрушения при растяжении с одновременным увеличением временного сопротивления разрушению.

Применение метода ПР после 4 циклов позволяет повысить сопротивление пластической деформации при

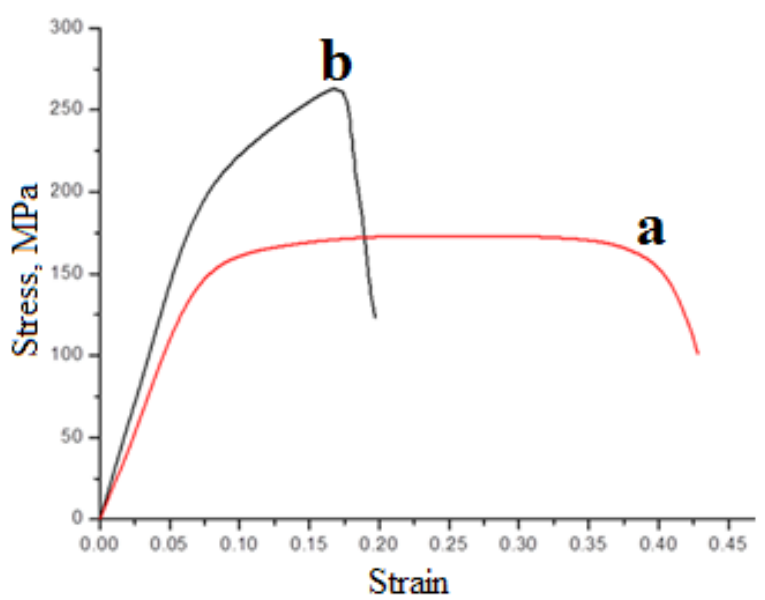

Рис. 4. Диаграмма условное напряжение - инженерная деформация для образцов алюминиевого сплава 1560 в состоянии поставки (a) и после 4 циклов ПР (b).

Fig. 4. Stress-strain curve: (a) - as-received condition sample, (b) treated sample. 
Таблица 1. Результаты испытаний на одноосное растяжение образцов из алюминиевого сплава 1560 в состоянии поставки и после четырех циклов ПР.

Table 1. Tensile tests results.

\begin{tabular}{|l|c|c|c|c|}
\hline \multicolumn{1}{|c|}{\begin{tabular}{c}
\multicolumn{1}{|c|}{ Образец } \\
Sample
\end{tabular}} & $\begin{array}{c}\sigma_{0.2}, \mathrm{Mna} \\
\sigma_{0.2}, \mathrm{MPa}\end{array}$ & $\begin{array}{c}\sigma_{B}, \mathrm{Mna} \\
\sigma_{B}, \mathrm{MPa}\end{array}$ & $\begin{array}{c}\delta_{B}, \% \\
\delta_{B}, \%\end{array}$ & $\psi, \%$ \\
\hline $\begin{array}{l}\text { сплав 1560 в состоянии поставки } \\
1560 \text { alloy as-received condition }\end{array}$ & $128.6 \pm 1.3$ & $173 \pm 1.7$ & $21 \pm 0.4$ & $42.8 \pm 0.8$ \\
\hline $\begin{array}{l}\text { сплав 1560 после четырех циклов ПР } \\
1560 \text { alloy treated соndition }\end{array}$ & $177.1 \pm 1.8$ & $263.2 \pm 2.6$ & $16.7 \pm 0.3$ & $19.7 \pm 0.4$ \\
\hline
\end{tabular}

скорости деформации $\left(1 \mathrm{c}^{-1}\right)$ алюминиевого сплава 1560 на 50\%. Эффект упрочнения в результате рифления при прессовании, сопровождается уменьшением пластичности, что является характерной чертой в поведении алюминиевых сплавов после ИПД.

Результаты исследований согласуются с данными описанными в $[16,17]$, где исследовали механических свойств алюминиевого сплава 1560 после обработки ИПД при РКУП. Авторами показано увеличение предела прочности образца после обработки в 2.1 и в 1.3 раза по сравнению с исходными литыми и прокатными образцами, соответственно. Значения микротвердости образцов после РКУП в работах $[16,18]$ находятся в диапазоне 105-135 HV, что соответствует значениям, полученным в данной работе.

\section{5. Заключение}

Проведено исследование влияния модификации зеренной структуры тонколистового проката алюминиевого сплава 1560 при четырехкратном прессовании с рифлением на деформирование сплава при растяжении и микротвердость. Установлено, что после 4 циклов прессования с рифлением листового проката 1.5 мм толщины достигнуто 52\% повышение значения величин временного сопротивления разрушению и $38 \%$ условного предела текучести, при испытаниях на одноосное растяжение в квазистатических условиях со скоростью деформации $1 \mathrm{c}^{-1}$. Достигнуто повышение значения величины микротвердости в образцах из листового проката алюминиевого сплава 1560 после обработки ПР. Впервые изучена зеренная структура, сформировавшаяся в результате обработки ПР в образцах из алюминиевого сплава 1560. Установлено, что эффект упрочнения исследуемого сплава сопровождается относительным уменьшением деформации на пределе прочности с 21\% в образце из сплава 1560 в состоянии поставки до 16.7\% в образце из сплава 1560 после ПР. Полученные данные согласуются с экспериментальными данными о повышении прочностных характеристик объемного проката сплава 1560, при использование других методов ИПД, например, равноканального углового прессования (РКУП) [16, 17, 18].
Таким образом, метод ПР представляет интерес для практического применения, поскольку позволяет дополнительно повышать прочностные характеристики листового проката популярного конструкционного алюминиевого сплава 1560 (АМг6). Sarkari Khorrami с соавторами было показано, что пластины из алюминиевых сплавов после РП могут быть соединены методом сварки трением без потери прочностных свойств в области соединения [19].

Благодарность/Aknowledgements. Исследования проведены с использованием оборудования иентра коллективного пользования "Аналитический иентр геохимии природных систем» Национального исследовательского Томского государственного университета. Работа поддержана частично Министерством образования и науки РФ (Госконтракт №2014/223, проект № 1943), проектом Фонда Д.И. Менделеева НИ ТГУ. Авторы благодарят за финансовую поддержку исследований.

Investigations were carried out using the equipment center for collective use "Analytical Center Geochemistry of Natural Systems" Tomsk State University. This work is supported by Ministry of Sciences and Education of Russian Federation (State task №2014/223, project № 1943), also “The Tomsk State University Academic D.I. Mendeleev Fund Program". The authors are grateful for the support of this research.

\section{Литература/References}

1. R.Z. Valiev. Russian nanotechnologies. 1, 208-216 (2006). (In Russian) [Р.3. Валиев. Российские нанотехнологии. 1, 208-216 (2006).]

2. R.R. Mulyukov. Russian nanotecnologies 2(7-8), 38-53 (2007). (In Russian) [P.P. Мулюков. Российские нанотехнологии. 2(7-8), 38-53 (2007).]

3. V.A. Skripnyak, E.G. Skripnyak, V.V. Skripnyak, A.A. Kozulin. Izvestiya vysshih uchebnyh zavedenij. Fizika. 55(9-3), 109-113 (2012). (In Russian) [B.А. Скрипняк, Е.Г. Скрипняк, А.А. Козулин, В.В. Скрипняк. Известия высших учебных заведений. Физика. 55(9-3), 109-113 (2012).] 
4. M.V. Markushev. Letters on Materials. 1(1), 36-42 (2011). (In Russian) [M.B. Маркушев. Письма о материалах. 1(1), 36-42 (2011).]

5. A.A. Kozulyn, V.A. Skripnyak, V.V. Skripnyak, V. A. Krasnoveikin, A.K. Karavatskii. Russian Physics Journal. 57(9), 1261-1267 (2015).

6. A.A. Nazarova, S.V. Dmitriev, Y.A. Baimova, R.R. Mulyukov, A.A. Nazarov. The physics of metals and metallography. 111(5), 513-519 (2011).

7. D.H. Shin, J. Park, Y. Kim, K. Park. Mater. Sci. Eng. 328, 98-103 (2002).

8. A. Krishnaiah, U. Chakkingal, P. Venugopal. Scripta Mater. 52, 1229-1233 (2005).

9. B. Ratna Sunil, A. Anil Kumar, T.S. Sampath Kumar, Uday Chakkingal. Mater. Sci. Eng. 33, 1607-1615 (2013).

10. A. Thirugnanam, T.S. Sampath Kumar, Uday Chakkingal. Mater. Sci. Eng. 30(1), 203-208 (2010).

11. A. Krishnaiah, U. Chakkingal, P. Venugopal. Mater. Sci. Eng. 410-411, 337-340 (2005).

12. Y. Saito, H. Utsunomiya, H. Suzuki, T. Sakai. Scripta Mater. 42, 1139-1144 (2000).

13. A. Shirdel, A. Khajeh, M.M. Moshksar. Mater. Design. 31, 946-950 (2010).
14. E.V. Avtokratova, O.E. Mukhametdinova, O.S. Sitdikov, M.V. Markushev. Letters on materials. 5(2), 129132 (2015). (In Russian) [Е.В. Автократова, О.Э. Мухаметдинова, О.Ш. Ситдиков, М.В. Маркушев. Письма о материалах. 5(2), 129-132 (2015).]

15. F. Khodabakhshi, M. Haghshenas, H. Eskandari, B. Koohbor. Mater. Sci. Eng. 636(11), 331-339 (2015).

16. A.A. Kozulin, V.A. Krasnoveikin, V.A. Skripnyak, et al. Sovrem. Probl. Nauki Obrazovan. 6, 888 (2013). (In Russian) [А.А. Козулин, В.А. Красновейкин, В.В. Скрипняк, Б.В. Хандаев, Ю.В. Ли. Современные проблемы науки и образования. 6, 888 (2013).]

17. V.N. Chuvil'deev, M.Yu. Gryaznov, V.I. Kopylov, A.N. Sysoev, B.V. Ovsyannikov, A.A. Flyagin. Vestnik Nizhegorodskogo universiteta im N.I. Lobachevskogo. 4, 35-42 (2008). (In Russian) [В.Н. Чувильдеев, М.Ю. Грязнов, В.И. Копылов, А.Н. Сысоев, Б.В. Овсянников, А.А. Флягин. Вестник Нижегородского университета им. Н.И. Лобачевского. 4, 35-42 (2008).]

18. M.V. Markushev, M.Yu. Murashkin Mater. Sci. Eng. 367(1-2), 234-242 (2004).

19. M. Sarkari Khorrami, M. Kazeminezhad, A.H. Kokabi. Materials \& Design. 45, 222-227 (2013). 